\title{
Research on the Ergonomic Adaptability of Electric Racing Vehicle
}

\author{
Zhang Dongjian $^{1}$, Ma Qihua ${ }^{1}$ and Tao Chao ${ }^{1}$ \\ ${ }^{1}$ Shanghai University of Engineering Science, School of Automotive Engineering, \\ Shanghai, 201620, China \\ 2367644811@qq.com,mqh0386@163.com
}

\begin{abstract}
This study shows that the electric racing vehicle (ERV) is still not well enough adapted for human beings, especially in the part of seat, steering wheel, visibility and accessible range. When designing ERV ergonomically, the virtual reality (VR) technique is as important as the real experience. Moreover, exacting contemporary economic and ecological requirements also mean that the cost and the energy consumption of the production cycle must be modified and reduced to a minimum. The present authors offer a new method of optimization of the ergonomic adaptability for ERV where the interiors and visibility are evaluated by designers and engineers, with interrelated functional links using UGS human builder module. This study uses anthropometric reference data for drivers from all previous racing drivers of the SUES. The objective was, taking into account the ERV interior height and width limitations, to accommodate the largest range of anthropometric dimensions by using the fifth-percentile woman, which was accomplished using a new method for model accommodation optimization. Furthermore, comfort, convenience, visibility and accessibility must be assured. Meanwhile, the robustness analysis also is applied to test the comfort of the ERV. After the robustness analysis of the new frame, the driver has enough comfort, convenience, visibility and accessible range of hands. By applying the suggested method and the data of new frame acquired, an optimum space for drivers was obtained. The space for the angle between the eyespot and the rear mirror is 12 deg relative to the vertical, and the horizontal angle is 29 deg, which meets the requirements of the basic visibility.
\end{abstract}

Keywords: virtual reality; ergonomic adaptability; UGS; optimization; robustness analysis

\section{Introduction}

During the last few years the ergonomics has been applied to the development of complex products, especially in automotive industry. This application successfully comes together from technological development and increased customer requirements of operational convenience, ride comfort and safety [1]. Nowadays, all manufacturers and suppliers already have regarded ergonomics as an important aspect of vehicle design. Therefore, more attentions have been paid to make sure that drivers and passengers can be best situated in their most convenient position to have a full control based on their functions.

During the late 1990s, in accordance with the "people-oriented" design concept, the world's major automobile companies pay more attentions to the application of ergonomics, which are used to improve the accessibility, visibility, comfort and safety of the vehicle. With the help of these techniques, the accessibility of pedals, steering wheel and general seating position can be evaluated, but more complex problems, such as the accessibility of certain controls, are difficult to solve [2-3]. 
The rapidly emerging technology of virtual ergonomics facilitates designers and engineers to overcome those issues by enabling the simulation of human interaction with a product or system from the initial stages of the design process, such as Finite Element Method (FEM) and Design of Experiments (DOE) approach [4]. In order to meet the global competition, automobiles manufactures have speed up the time to market new cars. In recent years, automotive designers are making an ever-increasing use of Virtual Reality (VR) techniques in order to validate their design during various phases of product development. Virtual ergonomic solutions bridge the gap between functional design and design for human comfort, productivity and safety [5-6]. By means of simulation in a virtual environment (VE), it is feasible to verify the performance of a human in context of a vehicle before it actually exists. Thus, it is possible to comprehend products centered on humans, suitable for various sizes and shapes, and to assess design based on ergonomic factors before building physical prototypes. The simulation will permit improvements in vehicle design relating to positioning, comfort, visibility, and access to controls from users of different sizes [7-8].

The progress of ergonomics in China has a considerable distance from the developed countries. But with the development of China's science, technology and economy, people's requirements on working conditions and quality of life are also improved gradually, so ergonomics characteristics of products will be paid more and more attentions [9].

Evaluation of the adaptability is a complex structured task, which directly affects the safety and comfort of the vehicle [10]. However comfort and safety can not be provided by adding the protection device alone in the ERV, which should be essentially designed not only by the dimensions of seat, including thigh width, lower-leg length, foot length, upper-arm length, forearm length, and hand length, but also by the angles between them[11-13]. In order to improve the accuracy of evaluation of the adaptability, automobile manufacturers must take into account the ergonomic requirements and constraints. In the past 10 years, with the development of automobile Digital Mock-up (DMU) and the virtual human model, the simulation of the driver and passengers are the extended in the early stages of the life cycle [14]. Currently, the evaluation of the adaptability tends to focus on humanity and comfort gradually, so the ergonomics in automobile general layout design occupies an increasingly important position.

The major aspects of ergonomics considered in this research are: comfort, convenience, vision, and robustness. Human Builder module of UGS was used to develop the accessibility analysis of driver, such as critical clearance distances and driving visibility analysis. Meanwhile, the study is aimed to design an evaluation system for the comfort of ERV through the robustness analysis in all previous frames.

\section{Model Development and Optimization}

\subsection{Comfort Analysis and Optimization}

The ERV studied in this article was shown in Figure 1. The fifth-percentile woman was used for predicting the ergonomics. The SgRP, AHP, L11, H17, A18 and w9 dimensions are shown in Figure 2. Where derived from the driver's actual sitting posture and frame data (Table 1 and 2). 


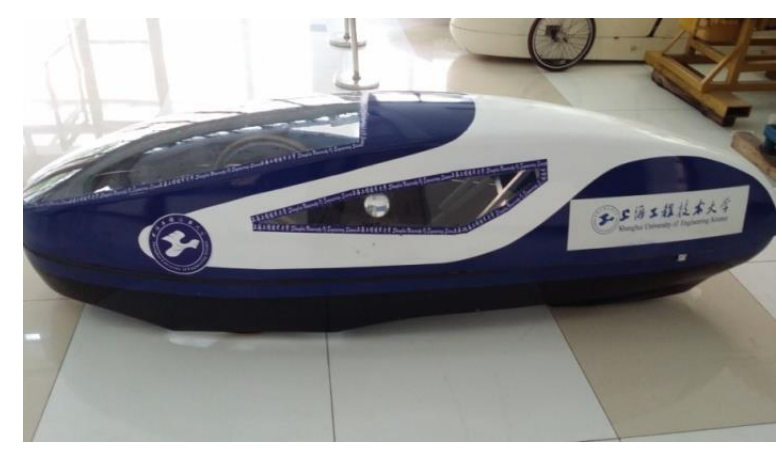

Figure 1. The Real Model of ERV
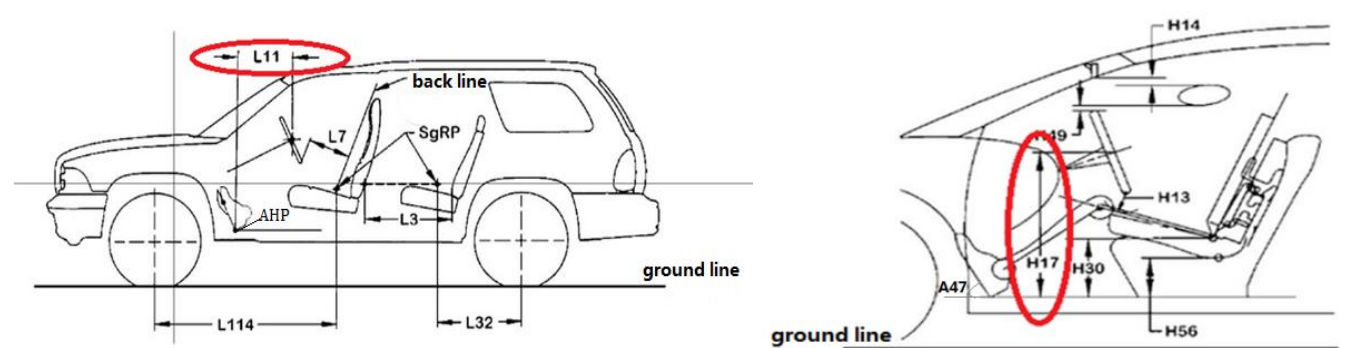

a) The distance from heel-point to steering wheel center b) The height of steering wheel center to AHP
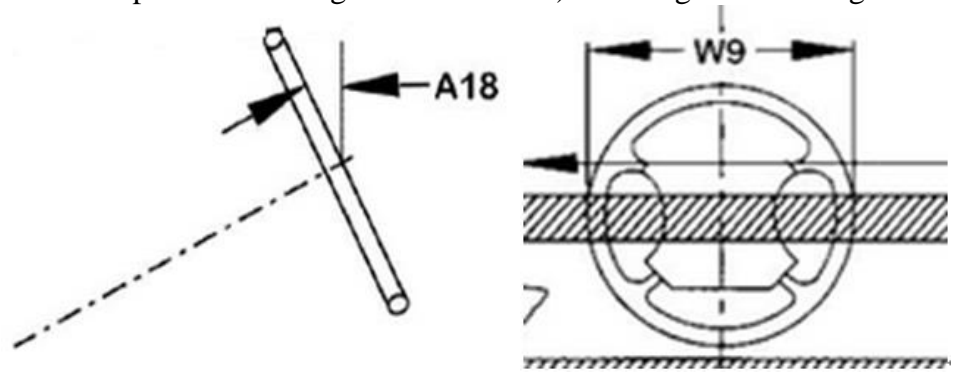

c) Steering wheel angle

d) Maximum diameter of steering wheel

Figure 2. Important Angle and Dimensions of the ERV

Table 1. Initial Sitting Position Data

\begin{tabular}{|c|c|c|c|c|c|c|c|}
\hline \multicolumn{3}{|c|}{ SgRP point } & \multicolumn{3}{c|}{ AHP point } & \multicolumn{2}{c|}{ sitting position data } \\
\hline $\mathrm{X}$ & $\mathrm{Y}$ & $\mathrm{Z}$ & $\mathrm{X}$ & $\mathrm{Y}$ & $\mathrm{Z}$ & $\mathrm{A} 40$ & A27 \\
\hline $740 \mathrm{~mm}$ & $0 \mathrm{~mm}$ & $70 \mathrm{~mm}$ & $70 \mathrm{~mm}$ & $140 \mathrm{~mm}$ & $0 \mathrm{~mm}$ & $40^{\circ}$ & $15^{\circ}$ \\
\hline
\end{tabular}

Table 2. Initial Vehicle Steering Wheel and Pedal Angle Data

\begin{tabular}{|c|c|c|c|c|}
\hline \multicolumn{4}{|c|}{ vehicle steering wheel } & pedal angle \\
\hline L11 & H17 & A18 & W9 & A47 \\
\hline $620 \mathrm{~mm}$ & $300 \mathrm{~mm}$ & $30^{\circ}$ & $265 \mathrm{~mm}$ & $85^{\circ}$ \\
\hline
\end{tabular}




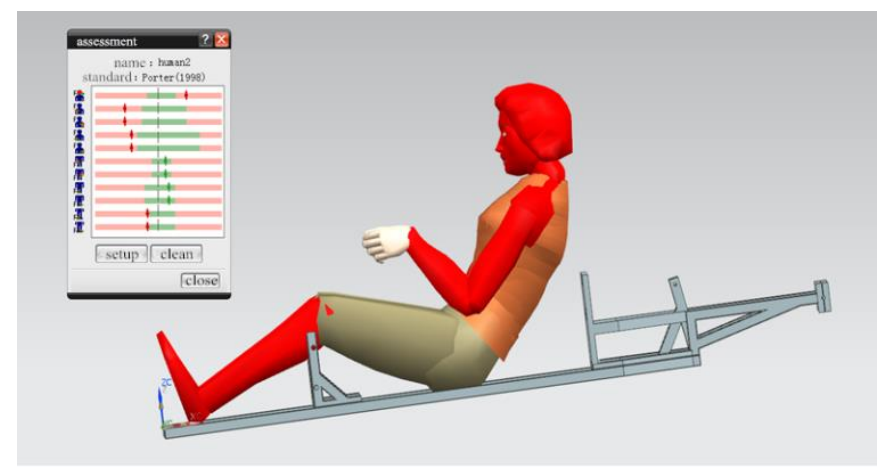

Figure 3. Driving Posture Prediction

The simulated results are shown in Figure 3, which can be concluded that the virtual driver was not in her comfort zone based on following reasons:

1) The driver's head is uncomfortable in the red area due to the baffle, which needs to be optimized.

2) The driver's shoulder and arms were shrinked in the chest which caused a discomfort for the driver due to the steering position constraints. It is reasonable to optimize the position of the steering wheel.

3) The positions of foot pedals have restricted the driver's lower legs and feet, resulting a uncomfortable long distance between AHP point and SgRP point and small angle between feet and lower legs.

After multiple adjustments and repeated experiments, the seating, steering wheel and other new data are given in the frame, as shown in the following table 3 and 4.

Table 3. Comparison of Seating Data

\begin{tabular}{|c|c|c|c|c|c|c|c|}
\hline & \multicolumn{2}{|c|}{ SgRP(mm) } & \multicolumn{3}{c|}{ AHP(mm) } & \multirow{2}{*}{ A40 $\left(^{\circ}\right)$} & A27( $\left(^{\circ}\right)$ \\
\hline & L31 & H70 & L8 & H8 & W20 & & \\
\hline Original frame & 740 & 0 & 70 & 0 & 140 & 40 & 15 \\
\hline New frame & 650 & 70 & 0 & 70 & 100 & 60 & 0 \\
\hline
\end{tabular}

Table 4. Comparison of the steering wheel and pedal data

\begin{tabular}{|c|c|c|c|c|c|}
\hline & L11 $(\mathrm{mm})$ & H17 $(\mathrm{mm})$ & A18 $(\mathrm{mm})$ & W9 $(\mathrm{mm})$ & A47( $\left(^{\circ}\right)$ \\
\hline Original frame & 620 & 300 & 30 & 265 & 85 \\
\hline New frame & 470 & 400 & 20 & 265 & 65 \\
\hline
\end{tabular}

The same fifth-percentile woman model was used for comparison. It is obvious that under new frame the driver's driving space becomes larger, the distance from heel-point to steering wheel center (L11) is shortened by $150 \mathrm{~mm}$, the height of steering wheel center to AHP (H17) is increased by $100 \mathrm{~mm}$, the steering wheel angle (A18) is decreased by 10 $\mathrm{deg}$, and maximum diameter of steering wheel (W9) is remained same size. The results show that all the critical joints are in comfort positions except head and neck (shown in Figure 4 (a)). 


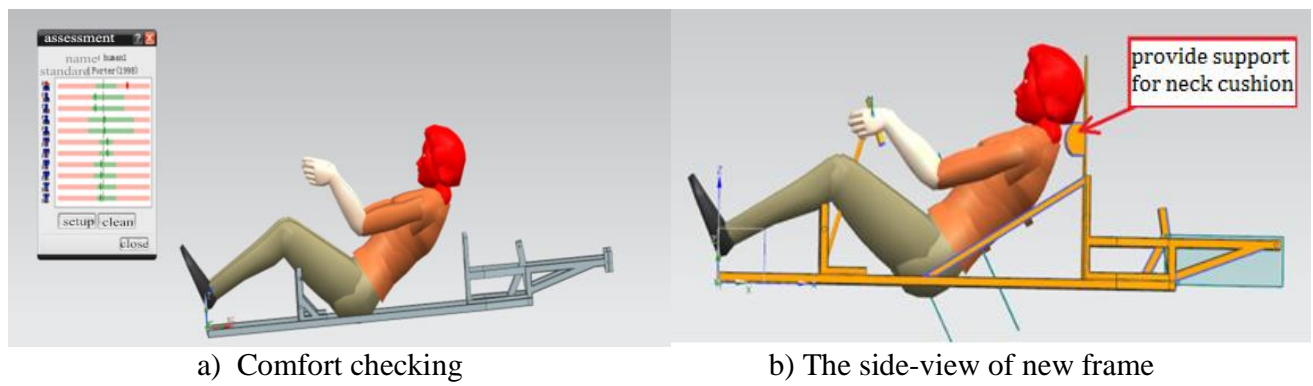

a) Comfort checking

b) The side-view of new frame

Figure 4. The Comfort Checking of New Frame

Subsequently, a variety of design schemes were added to increase comfort. For example: the front fork of the frame was moved forward, and the transverse stiffeners were removed to make it easy for drivers to getting on and off the cabin of the ERV (see Figure 4 (b)).

\subsection{Convenience Analysis and Improvement}

Convenience of getting on and off of the ERV is vital, and the comfort experience of driving need rely on some additional design to meet the requirements of the driver, such as the ladder in large engineering vehicles, boarding pedal for SUVs. Ordinary SUV door below pedal, and detachable steering wheel for formula 1 racing car.,

The study on the ERV frame will be infeasible before a series of experiments and changes; it is not conducive to the experience of driver comfort, especially in the getting off and up. In the middle of the experiment, the researcher invited a live model to sit in the ERV cabin of without modifications and steering wheel pasted in the belly of the driver tightly. Since the steering wheel is prominent with ribs and fork height, the driver's leg is extremely inconvenient.

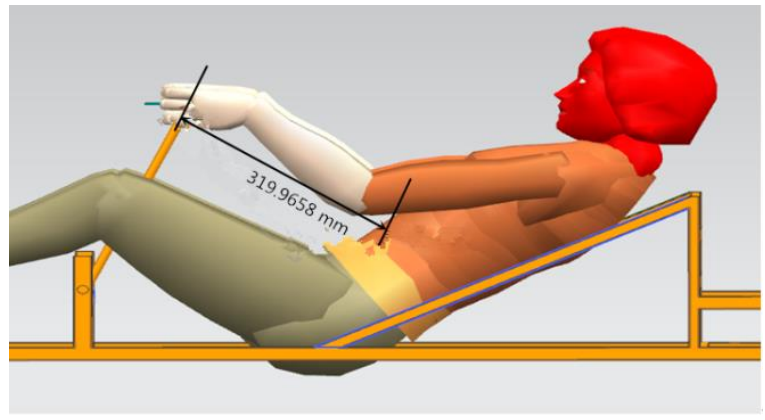

\section{Figure 5. Distance from the Steering Wheel Center to the Dummy Abdomen}

As shown in Figure 5, the distance of the steering wheel and dummy abdomen is about $32 \mathrm{~cm}$. After putting steering wheel center away from dummy abdomen, not only dummy elbow and shoulder comfort are greatly improved, but also legs are far away from the steering wheel. Thus legs are convenient in the frame and dummy sitting comfort is also improved.

The second advantage of the new frame is lied on the easy access for the driver. In the former model, the driver need other's help to get out of the driving cabin because her legs are stuck between the front forks. But after added oblique backrest frame which happens to play a role in the handle, driving members can hold hands in the back of the two sides of the ramp to support from their own while the legs pulled out of the frame.

Finally, the cross bar on the front fork is removed without affecting the frame strength, so the driver's legs can be pulled in and out of the frame freely. The front fork of the new 
frame need not add extra transverse stiffeners and also facilitate the driver to get on and off easily.

\subsection{Vision Checking}

In the UG, eyespots of E1 and E2 can be found by the dummy editing body function, coordinate respectively $(946.9838,32.577,553.2959)$ and $(946.9838,-32.5769$, 553.2959).

The distance from E1, E2 to P is $104 \mathrm{~mm}$, and the distance between E1 and E2 is $65 \mathrm{~mm}$. The coordinate data from the two point of the left and right can be calculated, so the $\mathrm{P}$ coordinate is $(1045.7738,553.2959)$. Then through the GB11562-94 standard of the backrest angle, V coordinate were calculated as V1 (976, -5, 509), V2 (976, -5, 443).

Based on Ergonomics in the horizontal direction, the eye comfortable rotation angle is 15 degrees and in the vertical direction also. In the UG software, the coordinate of measured vehicle shell top left corner point is $(225.6914,364.6272,597.7717)$, the coordinate of vehicle shell bottom left corner of the inflection angle is (236.4676, 303.1649 , 305.4636). As shown in the Figure 6(a), Left eyespot as the origin of coordinates, the vertical angle of eyespot on the $X O Z$ plane, this inclined upper at an angle of $17.198 \mathrm{deg}$ and down $22.218 \mathrm{deg}$, so the driver's vision is in a comfortable range in the vertical direction. Calculation of horizontal angle of eyespot on the $\mathrm{XOZ}$ plane, relative to the top left corner of the horizontal angle of degree $28.838 \mathrm{deg}$, relative to the bottom left corner of the horizontal angle of $25.259 \mathrm{deg}$ approximately, so the driver's vision is in a comfortable range in the horizontal direction. Based on the above calculation, the rotation angle of the right eye is also in the comfort range in accordance with the principle of symmetry. Also, there is a large part of the car under the shell is a transparent visual area. Therefore, the front view of the driver is very comprehensive and convenient for the driver's observation.

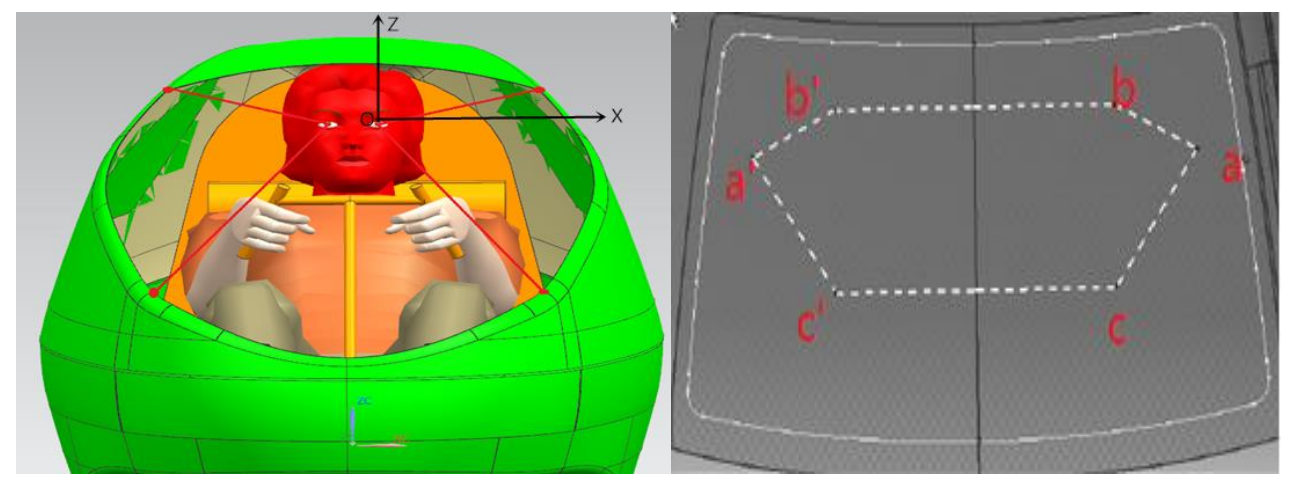

a) The angle between eyespot and front windshield b) The position of the A area on the front windshield

Figure 6. The Visibility of the Front Windshield

1) The reference point a is the E1 horizontal forward left deviation of $17 \mathrm{deg}$;

2) The reference point $b$ is the E1 vertical forward up deviation of $7 \mathrm{deg}$;

3) The reference point $\mathrm{c}$ is the E1 vertical forward down deviation of $5 \mathrm{deg}$;

4) on the other side of the vehicle's longitudinal symmetry plane, we should increase the auxiliary reference point a ', b', c '. As shown in Figure 6(b).

The front windshield was original in the driver's eye rotation angle of the comfort range. Therefore, smaller sweep area is in the driver's comfort range of vision. Since the vehicle is racing car, the car does not have the wiper. In order to cope with the competition in extreme weather, it is recommended to set up a simple wiper on the car shell. 
The national GB11562-1994 stipulates that at least 2.5 meters in the horizontal visual field should be shown in the driver rearview mirror and at least 4 meters for the passenger side. In addition, with the interior rearview mirror view the driver must see a width of at least 20 meters in horizontal road.

Because the ERV was set for one person and there's no interior rearview mirror, the rearview mirror view should show the area with 10 meters horizontal road and width of at least 2.5 meters, as shown in Figure 7.

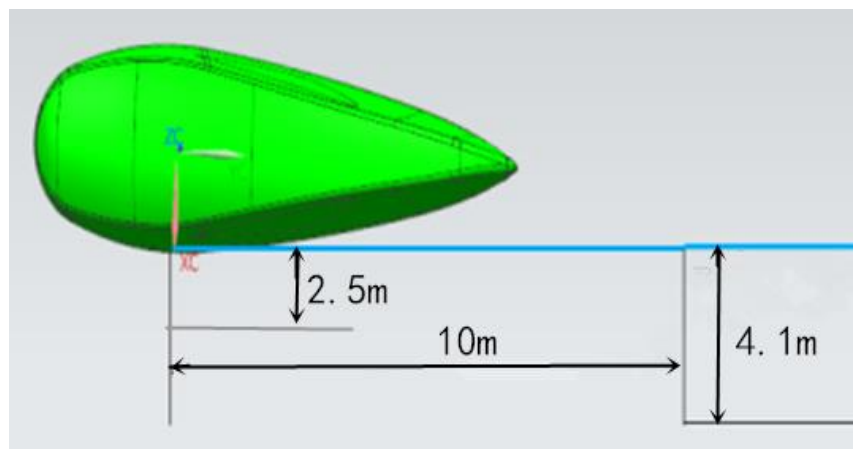

Figure 7. The Visibility of Rearview

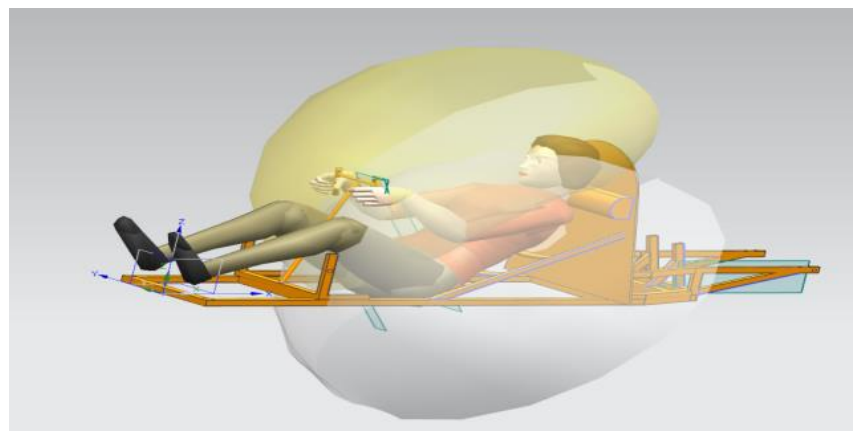

Figure 8. Hands Touch Area without Waist Rotation

\subsection{Accessible Range}

From in Figure 8, we can see clearly that the driver's hands can easily touch the steering wheel, and the back baffle plate area can be removed outside the car easily. The driver has good accessibility without rotating waist.

Under the condition that the waist can rotate, a larger range is accessible for driver, even some of the blind angles. Therefore, the driver's operation can be guaranteed. The key is the steering wheel's accessibility. When the accessibility of the steering wheel was achieved, so did the instruments mounted on the steering wheel. This design reduces the driver's sitting height, which ensures that the driver's head space will greater than the original one. Even if the driver does not wear a helmet, of the distance between head and roof is only $5 \mathrm{~cm}$. So under the new posture, driver is very convenient even wearing the helmet, to observe the road and reflective mirror easily.

\section{Application of Robustness Theory}

When looking at the relevant information of robustness found that the auto layout in virtual environment with robust design, the Professor of Antonio Lanzotti published, explained that through the use of UG software in the human body function simulation and data analysis of dummy posture found a particular position data in various states is 
reasonable for driver's comfort. In fact, this is the stability of robustness, so we can find the best car layout.

Since the robustness can be used in the design of the car comfort, it is feasible to find the robustness through data analysis, and design an evaluation system for the comfort of ERV through the research of the new frame.

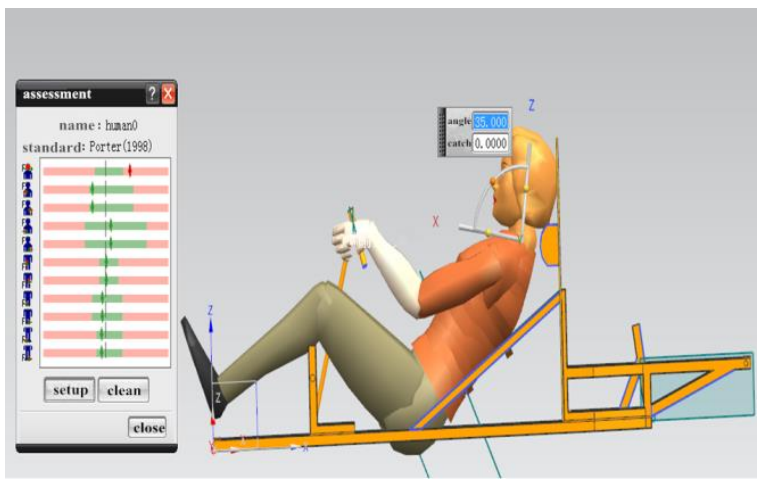

\section{Figure9. The Adjusted New Frame and Dummy Comfort}

First step, as is shown in Figure 9, due to the barrier of the rear baffle of the dummy's head, the head and trunk angle remained at about 35 degree, which is not in the comfort range. Assuming we can get rid of the baffle, the dummy head comfortable range is between -10 to 26 degree. But once over this range, the dummy's head will not be in the comfort range.

The second step, based on sitting comfort, is only to change the one part of the body that we will obtain the comfort range of other dummy body in other parts of the body remained motionless, as in table 5 .

Table 5. Dummy Limbs Maximum and Minimum Comfort Degree (unit: deg)

\begin{tabular}{|c|c|c|c|c|c|c|}
\hline & shoulder & elbow & thigh & calf & foot & head \\
\hline Original sitting posture & 30 & 60 & 69.035 & 67.225 & 3.3169 & 35 \\
\hline maximum & 80 & 94 & 81 & 81 & 10 & 26 \\
\hline minimum & 27 & 16 & 56 & 42 & -23 & -10 \\
\hline
\end{tabular}

In the third step, SgRP point location remained invariant when changes the posture. Researchers found that dummy shoulders and thighs comfort will be changed with the changing of the angle of femur and lumbar. So contrast to two sets of experiments, dummy femoral angle range is between -6.5 and 11 degree. According to maximum and minimum angles, adjusting the other limbs, we can get the comfort range of dummy limb. As shown in table 6 and 7. 
Table 6. Dummy Limbs Maximum and Minimum Comfort Range Based on Femoral Change (unit: deg)

\begin{tabular}{|c|c|c|c|c|c|c|c|}
\hline & femur & shoulder & elbow & thigh & calf & foot & head \\
\hline Original sitting posture & 9.6153 & 30 & 60 & 69.035 & 67.225 & 3.3169 & 35 \\
\hline maximum & 11 & 83 & 94 & 74 & 81 & 10 & 26 \\
\hline minimum & -6.5 & 28 & 16 & 50 & 42 & -23 & -10 \\
\hline
\end{tabular}

Table 7. Dummy Limbs Maximum and Minimum Comfort Range Based on Waist Change (unit: deg)

\begin{tabular}{|c|c|c|c|c|c|c|c|}
\hline & femur & shoulder & elbow & thigh & calf & foot & head \\
\hline Original sitting posture & 9.6153 & 30 & 60 & 69.035 & 67.225 & 3.3169 & 35 \\
\hline maximum & 84.540 & 123 & 94 & 50 & 81 & 10 & 26 \\
\hline minimum & -52.36 & 66 & 16 & 26 & 42 & -23 & -10 \\
\hline
\end{tabular}

From table 6 and 7, it can be observed that, regardless of posture changing of the dummy, the maximum and minimum comfortable degree of dummy's elbow, leg, foot and head were not changed. This phenomenon shows that the new frame has robust stability in comfort. It is worth mentioning that the experiment of the third step, although the dummy femoral angle is between -6.5 and $11 \mathrm{deg}$, the dummy body comfort is still within the range of comfort except head, as shown in Figure 10 and 11.

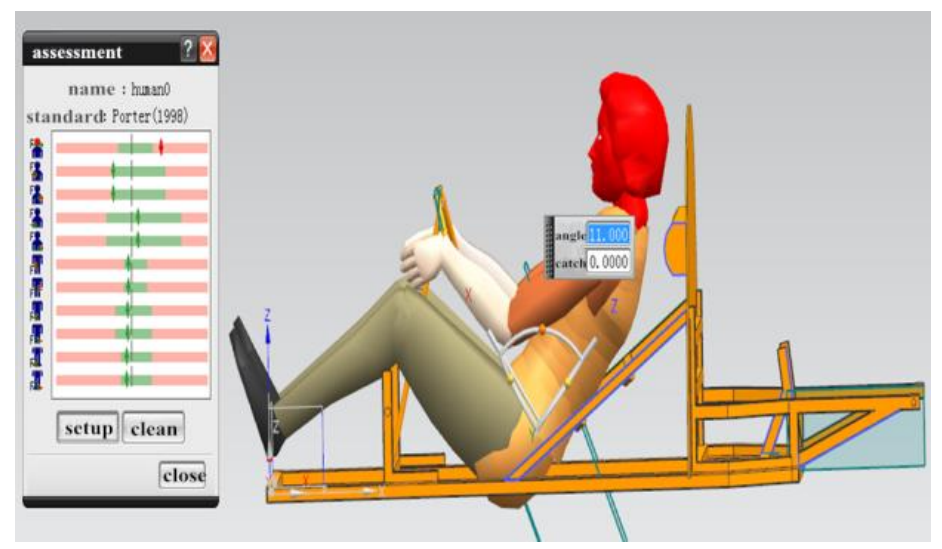

Figure 10. The Comfort Evaluation of the Femur at Maximum Degree 


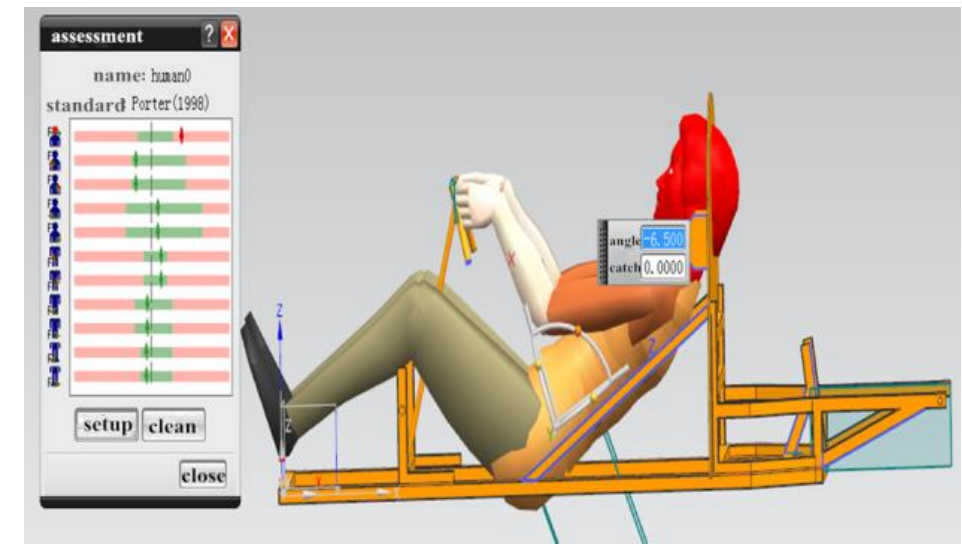

Figure11. The Comfort Evaluation of the Femur at Minimum Degree

The various parts of the body still can maintain a certain comfort even if gradually adjust the body angle in a certain range, at the same time, the robust asymptotic is fully revealed in this process. Experimental results show that the frame has two robust characteristics, which can satisfy the racing driver comfort requirements.

Through a series of experiments above for the new frame, the researcher uses the principle of robustness to establish a suitable evaluation system of comfort of the ERV. Due to the factors of the ERV and riding condition, the system has a certain distinction with ordinary passenger cars. Therefore, this system can be used in the evaluation of comfort of the ERV in the future.

According to the relevant literatures, the following test items and contents are developed in the evaluation of the driver's comfort level, shown in table 8.

Table 8. Experimental Results of the Joint Angle Value

\begin{tabular}{|c|c|c|c|}
\hline Joint angle & minimum & comfortable & maximum \\
\hline Head bending & -10 & 4 & 26 \\
\hline Shoulder bending & 26 & 45 & 94 \\
\hline elbow & 16 & 67 & 88 \\
\hline Angle between thigh and trunk & 64 & 80 & 81 \\
\hline Knee angle & 42 & 63 & 10 \\
\hline Foot & -23 & -2 & 94 \\
\hline
\end{tabular}

As shown in Table 8, the maximum and minimum joint angles are measured in the new frame. In order to research joint angle relation under the condition of different posture, researchers changed the angle of hip and waist and optimized the values of each joint angle, and then the optimal values of the other joint angles are obtained, as shown in Figure 12 and 13. 


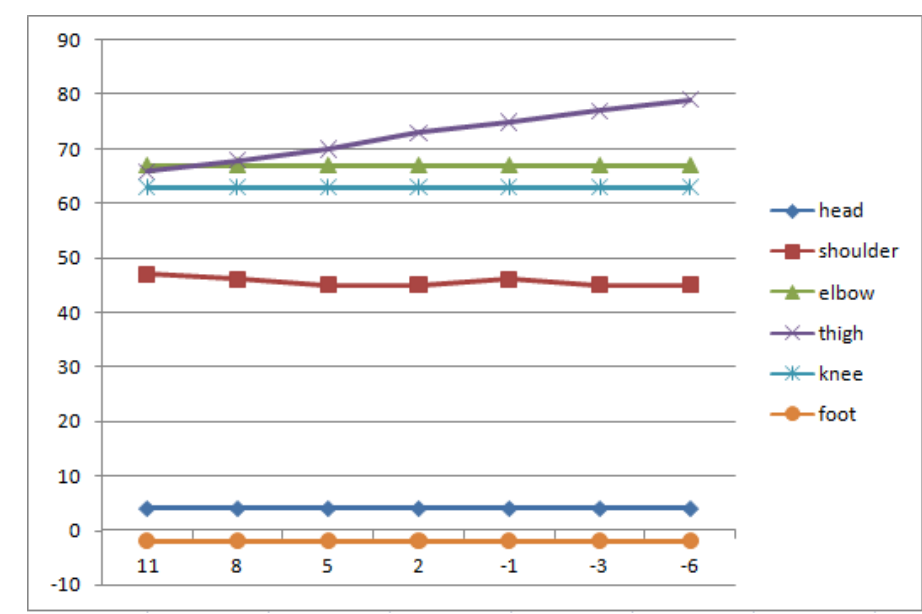

Figure12. Changes of Other Joints Caused by Crotch (unit: deg)

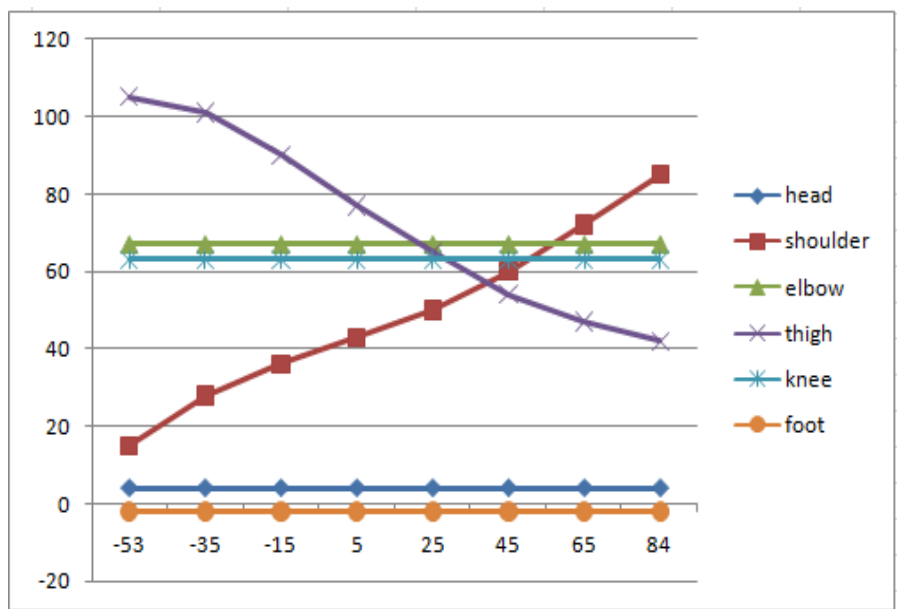

Figure13. Changes of Other Joints Caused by Waist (unit: deg)

Based on the comparison of figure 12 and 13, the angle of the driver's head, elbows, knees and feet is fixed, which reflects the robust stability of the frame. But the changes of shoulder and thigh angle will show inverse ratio with respect to the hip and waist.

Based on $Y=k X+b$, the basic principles of the evaluation system can be described as follows:

Formula of shoulder with the change of crotch:

$$
Y_{1}=8.5 X_{1}-388.5
$$

Formula of thigh with the change of crotch:

$$
Y_{1}=-1.3077 X_{2}+97.3077
$$

Formula of shoulder with the change of waist:

$$
Y_{2}=1.957 X_{3}-82.357
$$

Formula of thigh with the change of waist:

$$
Y_{2}=-2.1746 X_{4}+128.1746
$$

Where $Y_{1}$ and $Y_{2}$ refer to the degree of crotch and waist, respectively. X1、X2、X3、 $\mathrm{X} 4$ are the degree of the shoulder and thigh. To evaluate the comfort condition of a driver's driving posture, only need to input $\mathrm{X}, \mathrm{Y}, \mathrm{b}$ to the formula, and infer the value of $\mathrm{K}$. Its value is close to the value of $\mathrm{K}$ in the above four formulas, then it shows that the driver's sitting posture is more comfortable. 
Driver's field of vision in the game plays a very important role on car direction control of various kinds of road conditions, which must be guaranteed. After a number of driving experience and reference information, the vision is divided into three main vision ranges:

1) Central vision range: the angle is $2 \sim 5 \mathrm{deg}$, in the area of visual objects most clearly.

2) Instant vision range: the angle is $15 \mathrm{deg}$, that driver can see the object clearly in the limited time through the rotation of the eye.

3) Effective range of vision: the angle is $30 \mathrm{deg}$, that driver can see the object clearly with more attention through the rotation of the eye and head.

The appropriate target distance is $620 \mathrm{~mm}$, where it would be dizzy for the driver if lower than $250 \mathrm{~mm}$, and ambiguous view for more than $820 \mathrm{~mm}$. For rear view, the rearview mirror must be installed in the vertical comfortable rotation angle of eye due to the rear baffle limit, namely -15 to $15 \mathrm{deg}$. In the horizontal direction, the comfortable effect can be achieved by rotating the head, and the driver does not need to rotate too many angles for observing the rear view mirror.

\section{Conclusions}

It has been proved in this study that the ERV is still not fully adapted to human beings, especially in the part of the seat, steering wheel, visibility and accessible range. This study contributes both to design ergonomic adaptability optimization for the ERV and evaluation system for the comfort of ERV, by use of the robustness theory. Based on comfort assessment of the ERV, it was found that the car offers more comfort to the fifthpercentile woman, as the critical clearance distances are well within the safe limits as directed by SAE. Based on reach ability analysis, it can be said that all the controls, steering wheel, seat belt, etc. are within the reach of the driver's hand comfortably. The UGS human builder module is efficient in conducting the ergonomic analysis and the proposed improvements for the fifth-percentile woman were successfully implemented by the design team of ERV. The evaluation system based robustness theory to test the comfort for the interior as well as exterior of the car. The developed system provides a cost effective and time efficient solution for the ergonomic analysis in comparison to the physical prototype approach.

\section{Acknowledgments}

The Supports by the 12th five-year program connotation construction of Shanghai University of Engineering Science and National College Students' scientific research innovation project, moreover, Honda energy saving competition of China are gratefully acknowledged.

\section{References}

[1] D. P. Wood, "Safety and the car size effect: A Fundamental Explanation", Accident Analysis \& Prevention, vol. 9, no. 1, (1997), pp. 29-34.

[2] M. H. Abidi, A. M. El-Tamimi and A. M. Al-Ahmari, "Virtual Ergonomic Assessment of First Saudi Arabian Designed Car in a Semi-Immersive Environment", Proscenia Engineering, vol. 64, no. 6, (2013), pp. $622-631$.

[3] K. Mike, "A conceptual framework proposed to formalize the scientific investigation of automobile seat comfort", Applied Ergonomics, vol. 39, no. 1, (2008), pp. 15-27.

[4] E. Al-Momani and I. Rawabdeh, "An application of finite element method and design of experiments in the optimization of sheet metal blanking process", Jordan Journal of Mechanical and Industrial Engineering, vol. 2, no. 1, (2008), pp. 53-63.

[5] M. Sánchez, R. Garcia, A. Domingo, M. Camacho and M. Sebastián, "Application of a Virtual and Ergonomic Framework for an Industrial Light Vehicle Concept Assembly Process: A Case Report”, Procedia Engineering, vol. 132, no. 1, (2015), pp. 1077-1080. 
[6] B. Ola, "Comparison of car seats in low speed rear-end impacts using the BioRID dummy and the new neck injury criterion (NIC)", Accident Analysis \& Prevention, vol. 6, no. 9, (2000), pp. 32-36.

[7] S. Pheasant., "Body space Anthropometry", Ergonomics and the Design of Work, vol. 3, no. 1, (1996), pp. 11-13.

[8] A. Lanzotti, "Robust design of car packaging in virtual environment", International Journal for Interactive Design \& Manufacturing, vol. 2, no. 1, (2008), pp. 39-46.

[9] X.-Y. Peng, "Research and Realization of ergonomics evaluation method of automobile concept design", Chongqing Press, Chongqing University, (2004).

[10] M. M. Pérez Blanco, J. J. Blanco Fernández and P. F. Fernández, "Method for determining the adaptability of lining materials for vehicle components", EP. Patent 2006159, (2008).

[11] G.-P. Sun, "A digital mock-up visualization system capable of processing giga-scale CAD models", Computer-Aided Design, vol. 39, no. 2, (2007), pp. 133-141.

[12] D. Lämkull, L. Hanson and R. Örtengren, "A comparative study of digital human modeling simulation results and their outcomes in reality: A case study within manual assembly of automobiles", International Journal of Industrial Ergonomics, vol. 39, no. 2, (2009), pp. 428-441.

[13] M. M. Klarin, V. K. Spasojević-Brkić and Z. D. Sajfert, "Determination of passenger car interior space for foot controls accommodation", Proceedings of the Institution of Mechanical Engineers Part D Journal of Automobile Engineering, (2009).

[14] C. Vogt, C. Mergl and H. Bubb, "Interior layout design of passenger vehicles with RAMSIS", Human Factors \& Ergonomics in Manufacturing, vol. 15, no. 2, (2005), pp. 197-212.

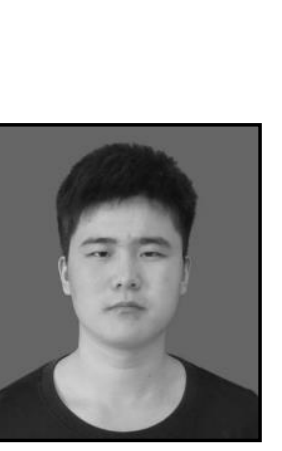

\section{Authors}

Zhang Dongjian, born in 1991, received Bachelor's degree in Huaiyin institute of technology, Huaiyin, China, in 2014. Since 2014, he has been a Master's degree candidate in Shanghai University of Engineering Science. His current interests include CAE analysis and Vehicle performance simulation.

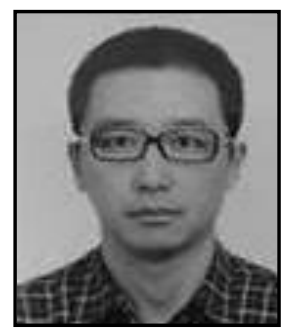

Ma Qihua, born in 1980. He is an associate professor in Shanghai University of Engineering Science. His main research interests are new energy and safety of automobile. 
International Journal of Hybrid Information Technology

Vol. 10, No. 8 (2017) 